\title{
LYSOSOMAL A-MANNOSIDASE
}

$\underline{\text { P. Heikinheimo }}^{1,2}$ R. Helland ${ }^{1}$ G. Schoehn ${ }^{3}$ R. Ravelli ${ }^{4}$ S. McSweeney ${ }^{3,4}$ O.-K. Tollersrud $^{1}$ E. Hough ${ }^{1,4}$

Department of Biochemistry Vatselankatu 2 UNIVERSITY OF TURKU FIN20014 FINLAND

${ }^{1}$ Departments of Chemistry and Medical Biochemistry, University of Troms $\varnothing$, N-9037 Troms $\varnothing$, Norway ${ }^{2}$ Department of Biochemistry, FIN-20014 University of Turku, Finland ${ }^{3}$ EMBL Grenoble Outstation, F-38042 Grenoble Cedex 9,

France ${ }^{4}$ ESRF, F-38043 Grenoble Cedex, France

Lysosomal $\alpha$-mannosidase (LAM) belongs to family 38 of glycoside hydrolysing enzymes. An inherited disease, $\alpha$-mannosidosis, is caused by the lack of lysosomal $\alpha$-mannosidase activity and accumulation of high-mannose chains, resulting in mental retardation, hearing loss and recurrent infections. During maturation and transport to lysosomes, the 999 residue bovine LAM (bLAM) is subject to both glycosylation and proteolytic processing. We have solved the structure of the mature bLAM at $2.7 \AA$ resolution. The electron micrograph maps of bLAM were used to identify the correct dimer from the crystallographic symmetry mates. The structure consists of an N-terminal $\alpha / \beta$ active site domain and 3 mostly $\beta$ domains. Sugar residues were built into assignable electron density at most of the glycosylation sites. Surface charge distribution, together with analysis of the chemical composition of the glycans allows prediction of areas important for lysosomal transport. One of the glycans is trapped into the structure and is involved in the dimer formation and possibly also in long range $\mathrm{pH}$ dependent domain movements. Even if the sequence identity is very low, the topology of the non-catalytic domains relates LAM to other carbohydrate processing enzymes such as $\beta$ galactosidase and maltose phosphorylase. A small all- $\beta$ domain in the $\mathrm{C}$-terminus is most likely regulatory since it has structure-based similarity to $\alpha$-amylase peptide inhibitors.

Keywords: GLYCOSIDE HYDROLYSING FAMILY 38, ALFAMANNOSIDASE, LYSOSOMAL TRANSPORT

\section{Acta Cryst. (2002). A58 (Supplement), C293 \\ STRUCTURAL AND FUNCTIONAL ANALYSES OF $\beta$-DOMAINS OF UvrB AND TRCF \\ N. Nakagawa ${ }^{1}$ M. Suzuki ${ }^{2}$ R. Masui $^{2}$ S. Kuramitsu ${ }^{1,2}$ \\ ${ }^{1}$ RIKEN Harima Institute Structurome Research Group 1-1-1, Kouto, Mikazuki-Cho, Sayo-Gun HYOGO 679-5148 JAPAN ${ }^{2}$ Osaka University}

In nucleotide excision repair system, UvrA-UvrB complex recognizes a wide range of DNA damages. Recently, we determined the crystal structure of UvrB from Thermus thermophilus HB8 (Nakagawa, et al. (1999) J. Biochem. 126, 986-990). Based on this structure and previous studies (Nakagawa, et al. (1997) J. Biol. Chem. 272, 22703-22713), we inferred that a discrete domain composed $\beta$-sheets ( $\beta$-domain) is the binding site of UvrA. Furthermore, sequence similar to the $\beta$-domain (UvrB- $\beta$ ) is found in transcription-repair coupling factor (TRCF). As TRCF also interacts with UvrA, this homologous region (TRCF- $\beta$ ) is considered as the UvrA-binding site. To prove this hypothesis and analyze the binding reaction in detail, we constructed the genes corresponding to UvrB- $\beta$ and TRCF- $\beta$. The purified $\beta$-domains retained their secondary structures up to $75^{\circ} \mathrm{C}$ at neutral $\mathrm{pH}$ and were found to exist as a monomer in solution. The crystal structure of UvrB- $\beta$ indicated that UvrB- $\beta$ keeps the same structure as $\beta$-domain within the intact protein. Surface plasmon resonance analysis using BIAcore showed binding of UvrA both to UvrB- $\beta$ and TRCF- $\beta$. Enzyme-coupled spectrophotometric assay indicated that $\beta$-domains have no effect on the ATPase activity of UvrA, although the activity was inhibited by the intact UvrB. Competition experiment between UvrB and $\beta$-domains with UvrA suggested that the binding region of UvrA to UvrB- $\beta$ and TRCF- $\beta$ were the same as the intact UvrB.

Keywords: DNA REPAIR THERMUS THERMOPHILUS DOMAIN
Acta Cryst. (2002). A58 (Supplement), C293

\section{STRUCTURE OF RAT BCKD KINASE: A PROTOTYPE FOR MITOCHONDRIAL PROTEIN KINASES}

M. Machius J. L. Chuang R. M. Wynn D. R. Tomchick D. T. Chuang

University of Texas Southwestern Medical Center At Dallas Biochemistry 5323 Harry Hines Blvd. L4.250 DALLAS TX 75390-9038 USA

Mitochondrial protein kinases (mPKs) are molecular switches that downregulate the oxidation of branched-chain $\alpha$-ketoacids and pyruvate. Elevated levels of these metabolites are implicated in disease states such as insulinresistant Type II diabetes, branched-chain ketoaciduria, and primary lactic acidosis. We report the three-dimensional structure of a member of the $\mathrm{mPK}$ family, rat branched-chain $\alpha$-ketoacid dehydrogenase kinase (BCK). BCK features a characteristic nucleotide-binding domain and a four-helix bundle domain. These two domains are reminiscent of modules found in protein histidine kinases (PHKs), which are involved in two-component signal transduction systems. Unlike PHKs, BCK dimerizes through direct interaction of two opposing nucleotide-binding domains. Nucleotide binding to BCK is uniquely mediated by both potassium and magnesium. Binding of ATP induces disorder-order transitions in a loop region at the nucleotide-binding site. These structural changes lead to the formation of a quadruple aromatic stack in the interface between the nucleotide-binding domain and the four-helix bundle domain, where they induce a movement of the top portion of two helices. Phosphotransfer induces further ordering of the loop region, effectively trapping the reaction product ADP, which explains product inhibition in mPKs. The BCK structure is a prototype for all $\mathrm{mPKs}$ and will provide a framework for structure-assisted inhibitor design for this family of kinases.

\section{Keywords: MITOCHONDRIAL PROTEIN KINASES BRANCHED CHAIN $\alpha$-KETOACID DEHYDROGENASE KINASE MAPLE SYRUP URINE DISEASE}

Acta Cryst. (2002). A58 (Supplement), C293

\section{THE CRYSTAL STRUCTURE OF THE CARBOXYTRANSFERASE SUBUNIT OF GLUTACONYL-CoA DECARBOXYLASE} K. S. Wendt ${ }^{1}$ I. Schall ${ }^{2}$ W. Buckel ${ }^{2}$ R. Huber ${ }^{1}$ U. Jacob

${ }^{1}$ Max-Planck Institut Fuer Biochemie Abt. Strukturforschung Max-Planck Institut Fuer Biochemie, Abt. Strukturforschung Am Klopferspitz 18 A MARTINSRIED 82152 GERMANY ${ }^{2}$ Laboratorium für Mikrobiologie, Fachbereich Biologie, Philipps-Universitaet, D-35032 Marburg

Glutaconyl-CoA decarboxylase from the Acidaminococcus fermentans is an ion pump, in which the free energy of decarboxylation drives the electrogenic transport of 1-2 $\mathrm{Na}^{+}$from the cytoplasm to the outside of the membrane in exchange to one proton1,2. It consists of four subunits of unknown stoichiometry. The carboxytransferase subunit a $(65 \mathrm{kDa})$ catalyzes the transfer of $\mathrm{CO}_{2}$ from glutaconyl-CoA to biotin, covalently attached to the $\mathrm{g}$ subunit. The decarboxylation of the carboxybiotin by the $b$ subunit, the ion pump, drives the $\mathrm{Na}+$ translocation. The $\mathrm{d}$ subunit is possibly a membrane-anchor for the a subunit. The carboxytransferase subunit could be crystallized in two different space groups: $P 4_{1} 2_{1} 2$ and $P 6_{4} 22$. The phase problem was solved with an MAD-experiment at DESY/Hamburg with crystals of space group $P 4_{1} 2_{1} 2$ of the selenomethionine labeled protein, additionally soaked with platinum terpyridine. Three platinum sites could be located and the program Shake \& Bake3 identified 27 of the 34 possible selenium sites. In the crystal a tetramer is formed by NCS and a crystallographic two fold axis. SIRAS-phasing and molecular averaging using the NCS operator led to an electron density sufficient for model building. The overall structure of the monomer is formed by two mostly parallel $\beta$ sheets flanked by a-helices on both sides of the sheet and is comparable to the Clp/crotonase-fold.

[1] Buckel, W. \& Semmler, R. Eur. J. Biochem. 136, 427-434 (1983).

[2] Buckel, W. Minireview, Biochim. Biophys. Acta 1505, 15-27 (2001).

[3] Weeks, C. M. \& Miller, R. J. Appl. Cryst. 32, 120-124 (1999).

Keywords: GLUTACONYL-COA DECARBOXYLASE, NA+TRANSLOCATING ION PUMP, COENZYME A 